\title{
Universal Schwinger Cocycles of Current Algebras in $(D+1)$-Dimensions: Geometry and Physics
}

\author{
Kazuyuki Fujii ${ }^{1}$ and Masaru Tanaka ${ }^{2}$ \\ 1 Department of Mathematics, Yokohama City University, 236 Japan \\ 2 Department of Physics, Kyushu University, 812 Japan
}

\begin{abstract}
We discuss the universal version of the Schwinger terms of current algebra (we call it the universal Schwinger cocycle) for $p=3$ (here $p$ denotes the class of the Schatten ideal $I_{p}$, which is related to the $(D+1)$ space-time dimensions by $p=(D+1) / 2)$ in detail, and give a conjecture of the general form of the cocycle for any $p$. We also discuss the infinite charge renormalizations, the highest weight vector and state vectors for $p=3$. Last, we give brief comments on the problems caused by the difficulties to construct the measure of infinite-dimensional Grassmann manifolds.
\end{abstract}

\section{Introduction}

In particle physics, current algebra has been introduced in the study of strong interactions. It was assumed that the time-component of a current generates a closed algebra in the classical level. More explicitly, we consider a Dirac field in $D+1$-dimension coupled to an external Yang-Mills field $A$. Let $G$ be a compact semi-simple Lie group and $g$ its algebra. The current is

$$
J^{i}(x)=\Psi^{\dagger}(x) \lambda^{i} \Psi(x) .
$$

We define

$$
J(f) \stackrel{\text { def }}{=} \int d x f^{i}(x) J^{i}(x)
$$

where $f(x)=f^{i}(x) \lambda^{i}: X \rightarrow g$, is a mapping valued in the Lie algebra.

This operator satisfies

$$
[J(f), J(g)]=J([f, g]) .
$$

But, in the quantum level, this relation is modified as follows:

$$
[J(f), J(g)]=J([f, g])+c(f, g ; A) .
$$

This $v(f, g ; A)$ is called the Schwinger term $[\mathrm{F}]$. This requires the representations of the Abelian extension Map $(X ; \underline{g})$ of $\operatorname{Map}(X ; \underline{g})$,

$$
(0 \rightarrow \operatorname{Map}(A ; C) \rightarrow \operatorname{Map}(X ; \underline{g}) \rightarrow \operatorname{Map}(X ; \underline{g}) \rightarrow 0) .
$$


In the present paper, we construct universal objects from the point of $K$-theory; $\mathrm{Gr}_{p}$, Det ${ }_{p}$, Det ${ }_{p}^{*}$, etc., for making the geometrical meaning of Schwinger terms and abelian extension. We discuss the two-cycle (universal Schwinger cocycle) for $p=3$ (the suffix $p$ denotes a suitable class of the Schatten ideal, which is related to the $(D+1)$ space-time dimensions by $p=(D+1) / 2)$, the two-cocyle for general $p$, the infinite charge renormalizations, and the highest weight vector. We also give brief comments for the measure of infinite-dimensional Grassmann manifolds.

Our results are the generalization of the work by Mickelsson and Rajeev [MR].

\section{Embedding of $\operatorname{Map}(X ; G)$ into the Infinite-Dimensional Group}

Let $X$ be the $D$-dimensional compact spin manifold, (for example, the $D$-dimensional torus), and define

$$
\operatorname{Map}(X: G)=\{g: X \rightarrow G, \text { smooth maps }\},
$$

then this space becomes a group by pointwise multiplication.

In the previous section, we considered the current algebra in the level of a Lie algebra. But, for our purpose, it is better to consider the same things in the level of a Lie group. Then, instead of $\operatorname{Map}(X ; G)$, we treat a larger group which acts on a Hilbert space.

Now we consider the Hilbert space $H$ consisting of free fermion fields $\Psi$ carrying a unitary representation $\rho$ of $G$.

Since a Dirac operator $D$ on $X$ has discrete eigenvalues, let $H_{+}$be the space of the eigenstates with positive eigenvalues of the operator $D$, and $H_{-}$the space of the eigenstates with its non-positive eigenvalues. Let the basis of each eigenspace of $D$ be

$$
\begin{array}{r}
\left\{e_{1}, e_{2}, \ldots\right\}: \text { orthogonal basis of } H_{+}, \\
\left\{e_{0}, e_{-1}, \ldots\right\} \text { :orthogonal basis of } H_{-} .
\end{array}
$$

Then $H=H_{+} \oplus H_{-}$. We define the sign operator $\varepsilon$,

$$
\varepsilon \stackrel{\text { def }}{=} \frac{D}{|D|} \text {. }
$$

(If $D$ has a zero eigenvalue, we set $\varepsilon=-1$.)

We define the operator $M(f): H \rightarrow H$ such that

$$
[M(f) \Psi](x) \stackrel{\text { def. }}{=} \rho(f(x)) \Psi(x) .
$$

$M(f)$ is decomposed as follows,

$$
M(f)=\left(\begin{array}{ll}
a & b \\
c & d
\end{array}\right),
$$

where

$$
\begin{cases}a: H_{+} \rightarrow H_{+}, & d: H_{-} \rightarrow H_{-} \\ b: H_{-} \rightarrow H_{+}, & c: H_{+} \rightarrow H_{-}\end{cases}
$$


We define the Schatten Ideal

$$
I_{2 p}=\left\{A \in B(H) \mid\|A\|_{2 p}=\left[\operatorname{tr}\left(A^{\dagger} A\right)^{p}\right]^{1 / 2 p}<\infty\right\},
$$

where $B(H)$ is the space of all bounded operators on $H[\mathrm{~S}],[\mathrm{C}]$.

We also define

$$
G L_{p} \stackrel{\text { def }}{=}\left\{A \in G L(H) \mid \operatorname{tr}[\varepsilon, A]^{2 p}<\infty\right] \quad(p=1,2, \ldots),
$$

where $G L(H)$ is the set of all invertible operators on $H$.

We note each $g \in G L_{p}$ can be written as

$$
g=\left(\begin{array}{ll}
a & b \\
c & d
\end{array}\right) ; \quad a, d \in \text { Fredholm and } b, c \in I_{2 p} .
$$

If $2 p>d$, there exists a continuous injective homomorphism [MR]

$$
M: \operatorname{Max}(X ; G) \subset G L_{p}
$$

\section{Properties of Generalized Determinant [S]}

We define the generalized determinant. For $A \in 1+I_{p}$,

$$
R_{p}(A)=-1+(1+A) \exp \left\{\sum_{j=1}^{p-1}(-1)^{j} \frac{A^{j}}{j}\right\} \in I_{1},
$$

and therefore $\operatorname{det}_{p} A$ is defined as

$$
\operatorname{det}_{p} A \stackrel{\text { def }}{=} \operatorname{det}\left(1+R_{p}(A)\right) .
$$

This form is a bit abstract. However, if $A$ satisfies the condition $\|A-1\|<1$, then we find $[\mathrm{MR}]$,

$$
\operatorname{det}_{p} A=\exp \operatorname{tr}\left\{(-1)^{p-1} \frac{(A-1)^{p}}{p}+(-1)^{p} \frac{(A-1)^{p+1}}{p+1}+\cdots\right\} .
$$

This determinant satisfies the following properties $\left(A, B \in 1+I_{p}\right)$ :

(i) $\mathrm{A}$ is invertible off $\operatorname{det}_{p} A \neq 0$.

(ii) If $A \in 1+I_{p-1}$, then

$$
\operatorname{det}_{p} A=\operatorname{det}_{p-1} A \cdot \exp \left[(-1)^{p-1} \operatorname{tr} \frac{(A-1)^{p-1}}{p-1}\right] .
$$

(iii) There exists a symmetric polynomial $\gamma_{p}(A, B)$ such that

$$
\operatorname{det}_{p} A B=e^{\gamma_{p}(A, B)} \operatorname{det}_{p} A \cdot \operatorname{det}_{p} B .
$$

We list first a few examples:

$$
\begin{aligned}
& \gamma_{1}(A, B) \equiv 0 \quad\left(A, B \in 1+I_{1}\right), \\
& \gamma_{2}(A, B)=-\operatorname{tr}(A-1)(B-1) \quad\left(A, B \in 1+I_{2}\right),
\end{aligned}
$$




$$
\begin{aligned}
\gamma_{3}(A, B)= & \operatorname{tr}\left\{\frac{1}{2}(A-1)(B-1)(A-1)(B-1)+(A-1)(B-1)(A-1)\right. \\
& +(B-1)(A-1)(B-1)\} \quad\left(A, B \in 1+I_{3}\right) .
\end{aligned}
$$

Now, if we define

then $[\mathrm{MR}]$,

$$
\omega_{p}(A, B) \stackrel{\text { def. }}{=} \operatorname{det}_{p} B \cdot e^{\gamma_{p}(A, B)},
$$

$$
\omega_{p}(A, B C)=\omega_{p}(A B, C) \cdot \omega_{p}(A, B),
$$

where $A, B, C \in 1+I_{p}$.

\section{Construction of Abelian Extension of $G L_{p}[\mathrm{MR}]$}

We define the subgroup $B_{p}$ of $G L_{p}$,

$$
B_{p} \stackrel{\text { def. }}{=}\left\{\left(\begin{array}{ll}
a & b \\
0 & d
\end{array}\right) \in G L_{p} \mid a, d \in \text { Fredholm, } b \in I_{2 p}\right\},
$$

and define the homogeneous space $G L_{p} / B_{p}$. This space is identified with the Grassmann manifold $\mathrm{Gr}_{p}$,

$$
\mathrm{Gr}_{p}=\left\{W \subset H \mid W=g \cdot H_{+}, g \in G L_{p}\right\} .
$$

We also define the orthogonal projections $\mathrm{pr}_{ \pm}$,

$$
\mathrm{pr}_{ \pm}: W \rightarrow H_{ \pm} \text {. }
$$

Since the diagonal block of $g \in G L_{p}$ is Fredholm, $\mathrm{pr}_{+}$is Fredholm. The off-diagonal block of $g$ is in the class of $I_{2 p}$, so $\mathrm{pr}_{-}$is in the class of $I_{2 p}$.

We set $G L^{p}$ as

$$
G L^{p} \stackrel{\text { def. }}{=} G L\left(H_{+}\right) \cap\left(1+I_{p}\right)
$$

where $G L\left(H_{+}\right)$is the set of all invertible operators on $H_{+}$.

We define a group

$$
\varepsilon_{p}=\left\{(g, q) \in G L_{p} \times G L\left(H_{+}\right) \mid a q^{-1}-1 \in I_{p}\right\},
$$

whose group multiplication is

$$
\left(g_{1}, q_{1}\right) \cdot\left(g_{2}, q_{2}\right)=\left(g_{1} g_{2}, q_{1} q_{2}\right) .
$$

The group $G L^{p}$ acts from the right on $\varepsilon_{p}$ by $(g, q) \cdot t=(g, q t)$, so we have $G L_{p}=\varepsilon_{p} / G L^{p}$. Since $B_{p}$ acts on $\varepsilon_{p}$ by $(g, q) \cdot k=(g k, q \alpha)$, where $k=\left(\begin{array}{ll}\alpha & \beta \\ 0 & \gamma\end{array}\right) \in B_{p}$, we can define
the Stiefel manifold

$$
\mathrm{St}_{p} \stackrel{\text { def. }}{=} \varepsilon_{p} / B_{p}
$$

which has the canonical projection $\pi: \mathrm{St}_{p} \rightarrow \mathrm{Gr}_{p}$.

Let $w=\left\{w_{1}, w_{2}, \ldots\right\}$ be the basis of $W \in \mathrm{Gr}_{p}$. Then

$$
\operatorname{pr}_{+}\left(w_{i}\right)=\sum_{j=1}^{\infty}\left(w_{+}\right)_{j i} e_{j}
$$


We call the basis $w$ admissible if $w_{+} \in 1+I_{p}$. Then we can show that every $W \in \mathrm{Gr}_{p}$ has an admissible basis.

From now on, we set

$$
w \stackrel{\text { def. }}{=}\left(\begin{array}{c}
w_{+} \\
w_{-}
\end{array}\right), \quad w_{ \pm}=\operatorname{pr}_{ \pm}(w)
$$

where $w_{+} \in 1+I_{p}$ and $w_{-} \in I_{2 p}$.

The right action of $t \in G L^{p}$ is the basis transformation $w_{i}^{\prime}=\sum_{j} w_{j} t_{j i}$.

This action of $G L^{p}$ on $\mathrm{St}_{p}$ is written shortely as

$$
\left(\begin{array}{c}
w_{+} \\
w_{-}
\end{array}\right) \mapsto\left(\begin{array}{c}
w_{+} t \\
w_{-} t
\end{array}\right)
$$

and induces the right action on $\mathrm{St}_{p} \times C$ as follows,

$$
(w, \lambda) \cdot t \stackrel{\text { def. }}{=}\left(w t, \lambda \omega_{p}\left(w_{+}, t\right)^{-1}\right) .
$$

Then we have the homogeneous space,

$$
\operatorname{Det}_{p} \stackrel{\text { def }}{=}\left(\mathrm{St}_{p} \times C\right) / G L^{p}
$$

which is the line bundle over the Grassmannian $\mathrm{Gr}_{p}$, whose projection is

$$
[(w, \lambda)] \mapsto \text { the space spanned by the basis }\left\{w_{1}, w_{2}, \ldots\right\} \text {. }
$$

Let $F=F(w)=\left(\begin{array}{ll}F_{11} & F_{12} \\ F_{21} & F_{22}\end{array}\right)$ be the linear operator in $H=H_{+} \oplus H_{-}$such that $\left.F\right|_{W}=+1,\left.F\right|_{W^{\perp}}=-1$, and $F^{2}=1$ on $H$, where $W$ is the plane determined by the basis $w=\left\{w_{i}\right\}$ (we can set $F=2 w\left(w^{\dagger} w\right)^{-1} w^{\dagger}-1$, especially $F=2 w w^{\dagger}-1$ for $\left.w^{\dagger} w=1\right)$.

We consider smooth functions $\alpha(g, q ; w)$ on $\varepsilon_{p} \times \mathrm{St}_{p}$ such that

$$
\frac{\alpha(g, q ; w t)}{\alpha(g, q ; w)}=\frac{\omega_{p}\left(w_{+}, t\right)}{\omega_{p}\left(\left(g w q^{-1}\right)_{+}, q t q^{-1}\right)}
$$

for $t \in G L^{p}$. A general solution of this equation is given by

$$
\alpha(g, q ; w)=f(g, q ; W) \frac{\operatorname{det}_{p} w_{+}}{\operatorname{det}_{p}\left(g w q^{-1}\right)_{+}} \cdot \frac{\operatorname{det}_{p} \frac{1}{2}\left(q^{-1} a\left(F_{11}+1\right)+q^{-1} b F_{21}\right)}{\operatorname{det}_{p} \frac{1}{2}\left(F_{11}+1\right)},
$$

where $f: \varepsilon_{p} \times \mathrm{Gr}_{p} \rightarrow C^{\times}$is an arbitrary smooth function [MR].

We define a group $\varepsilon_{p} \times \operatorname{Map}\left(\mathrm{Gr}_{p}, C^{\times}\right)$, whose group structure is defined by

$$
\begin{aligned}
\left(g_{1}, q_{1}, \mu_{1}\right)\left(g_{2}, q_{2}, \mu_{2}\right)= & \left(g_{1} g_{2}, q_{1} q_{2}, \mu_{1}\left(g_{2} \cdot F\right) \mu_{2}(F)\right. \\
& \left.\cdot \propto\left(g_{1}, q_{1} ; q_{2} w q_{2}^{-1}\right) \alpha\left(g_{2}, q_{2} ; w\right) \alpha\left(g_{1} g_{2}, q_{1} q_{2} ; w\right)^{-1}\right) .
\end{aligned}
$$

$\varepsilon_{p} \times \operatorname{Map}\left(\mathrm{Gr}_{p}, C^{\times}\right)$acts on $\operatorname{Det}_{p}$ by the formula

$$
(g, q, \mu) \cdot(w, \lambda)=\left(g w q^{-1}, \mu(\pi(w)) \cdot \lambda \cdot \alpha(g, q ; w)\right) .
$$


There is an Abelian extension $\widehat{G L_{p}}$ of $G L_{p}$ by $\operatorname{Map}\left(\mathrm{Gr}_{p}, C^{\times}\right)$,

$$
\widehat{G L_{p}}=\left(\varepsilon_{p} \times \operatorname{Map}\left(\mathrm{Gr}_{p}, C^{\times}\right)\right) / N_{p},
$$

where $N_{p}$ is the kernel of this action (the normal subgroup) consisting of elements $\left(1, q, \mu_{q}\right)$, where

$$
\begin{gathered}
\mu_{q}(w)=\alpha(1, q, w)^{-1} \cdot \omega_{p}\left(w_{+}, q^{-1}\right)^{-1}, \quad q \in G L^{p}, \\
\left(1 \rightarrow \operatorname{Map}\left(\mathrm{Gr}_{p}, C^{\times}\right) \rightarrow \widehat{G L_{p}} \rightarrow G L_{p} \rightarrow 1\right) .
\end{gathered}
$$

We note that if $p=1$, the above sequence is

$$
1 \rightarrow \operatorname{Map}\left(\mathrm{Gr}_{1}, C^{\times}\right) \rightarrow \widehat{G L_{1}} \rightarrow G L_{1} \rightarrow 1,
$$

which seems an Abelian extension but is reduced to a central extension [PS],

$$
1 \rightarrow C^{\times} \rightarrow \widehat{G L_{1}} \rightarrow G L_{1} \rightarrow 1 .
$$

Here we list various bundles constructed by [MR].

(i) $p>1$ :

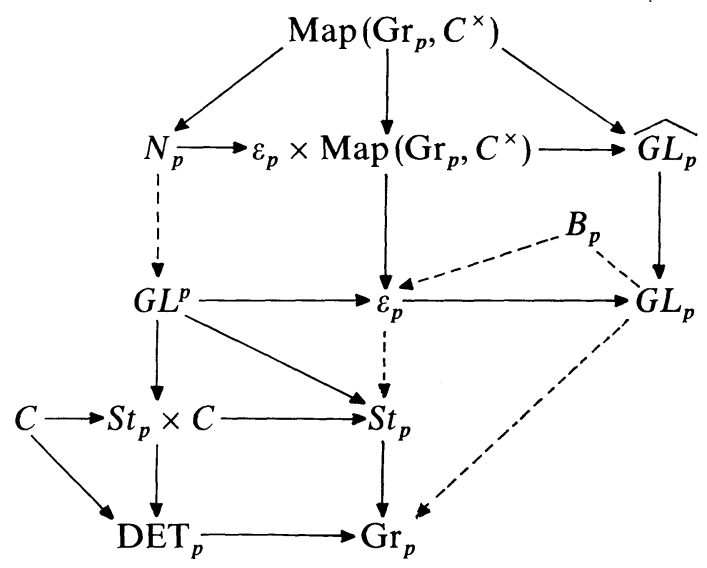

(ii) $p=1$ :

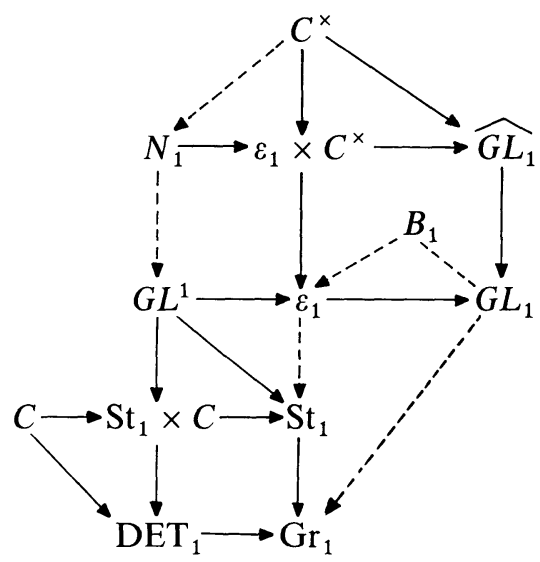


(Avoiding confusions, two kinds of lines are used, and they stand for the bundle maps.)

Near the unit element $g=1$, we can define the local section $\Gamma: \widehat{G L_{p}} \rightarrow G L_{p}$ by

$$
\Gamma(g)=(g, a, 1) \bmod N_{p} .
$$

The extension is in general determined by the two-cocycle. In this case, the two-cocycle is computed by

$$
\Gamma\left(g_{1}\right) \cdot \Gamma\left(g_{2}\right)=\Gamma\left(g_{1} g_{2}\right) \cdot\left(1,1, \xi\left(g_{1}, g_{2}\right)\right),
$$

where $\xi\left(g_{1}, g_{2}\right) \in \operatorname{Map}\left(G L_{p}, C^{\times}\right)$.

By the associativity, $\xi$ satisfies the following condition:

$$
\xi\left(g_{1}, g_{2} g_{3} ; \pi(\omega)\right) \cdot \xi\left(g_{2}, g_{3} ; \pi(\omega)\right)=\xi\left(g_{1} g_{2}, g_{3} ; \pi(\omega)\right) \cdot \xi\left(g_{1}, g_{2} ; \pi\left(g_{3} \omega\right)\right) \text {. }
$$

It is, in general, complicated to treat the group extension, but the corresponding Lie algebra is rather simpler. So we consider the infinitesimal version of the group extension,

$$
\left(0 \rightarrow \operatorname{Map}\left(\mathrm{Gr}_{p}, C\right) \rightarrow \widehat{g l_{p}} \rightarrow g l_{p} \rightarrow 0\right) .
$$

The Lie algebra $\widehat{g l_{p}}$ of $\widehat{G L_{p}}$ is equivalent to $g l_{p} \oplus \operatorname{Map}\left(\mathrm{Gr}_{p}, C\right)$ as a vector space, where $g l_{p}$ is the Lie algebra of $G L_{p}$.

The commutator in $g l_{p}$ is

$$
[(X, \mu),(Y, v)]=([X, Y], X \cdot v-Y \cdot \mu+\eta(X, Y ; F)),
$$

where $\eta$ is an antisymmetric bilinear form on $g l_{p}$ taking values in $\operatorname{Map}\left(\mathrm{Gr}_{p}, C\right)$ and $X \cdot v$ is a Lie derivative of a function $v$ on $\mathrm{Gr}_{p}$ to the direction of the vector field $X$ defined by the $G L_{p}$ action on $\mathrm{Gr}_{p}$.

From the Jacobi identity, we have

$$
\begin{aligned}
& \eta([X, Y], Z ; F)+\eta([Y, Z], X ; F)+\eta([Z, X], Y ; F) \\
& \quad-Z \cdot \eta(X, Y ; F)-X \cdot \eta(Y, Z ; F)-Y \cdot \eta(Z, X ; F)=0 .
\end{aligned}
$$

Now let's compute the two-cocycle. By Eq. (41), we have

$$
\begin{aligned}
& \left\{\Gamma\left(g_{1}\right) \Gamma\left(g_{2}\right) \Gamma\left(g_{1}^{-1}\right) \Gamma\left(g_{2}^{-1}\right)\right\}(w, \lambda) \\
& \quad=\left(g_{1}, g_{2} g_{1}^{-1} g_{2}^{-1}, a\left(g_{1}\right) a\left(g_{2}\right) a\left(g_{1}^{-1}\right) a\left(g_{2}^{-1}\right), \mu\right)(w, \lambda),
\end{aligned}
$$

where $\mu$ is

$$
\begin{aligned}
\mu(\pi(w))= & \alpha\left(g_{2}^{-1}, a\left(g_{2}^{-1}\right) ; w\right) \\
& \cdot \alpha\left(g_{1}^{-1}, a\left(g_{1}^{-1}\right) ; g_{2}^{-1} w a\left(g_{2}^{-1}\right)^{-1}\right) \\
& \cdot \alpha\left(g_{2}, a\left(g_{2}\right) ; g_{1}^{-1} g_{2}^{-1} w a\left(g_{2}^{-1}\right)^{-1} a\left(g_{1}^{-1}\right)^{-1}\right) \\
& \cdot \alpha\left(g_{1}, a\left(g_{1}\right) ; g_{2} g_{1}^{-1} g_{2}^{-1} w a\left(g_{2}^{-1}\right)^{-1} a\left(g_{1}^{-1}\right)^{-1} a\left(g_{2}\right)^{-1}\right) \\
& \cdot \alpha\left(g_{1} g_{2} g_{1}^{-1} g_{2}^{-1}, a\left(g_{1}\right) a\left(g_{2}\right) a\left(g_{1}^{-1}\right) a\left(g_{2}^{-1}\right) ; w\right)^{-1} .
\end{aligned}
$$

To obtain the two-cocycle $\eta_{p}(X, Y ; F)$, we set $g_{1}=e^{t X}$ and $g_{2}=e^{s Y}$, and compute

$$
\left.\frac{\partial^{2}}{\partial s \partial t} \mu(\pi(w))\right|_{s=t=0}=\eta_{p}(X, Y ; F)
$$


We can get the two-cocycle $\eta_{p}(X, Y ; F)$ with the suitable choice of $f(g, q ; W)$ in Eq. (40). (In is not easy to choose $f(g, q ; W)$ for each $p$.)

We must note that the calculations below are based on $\hat{U}_{p}$ (the unitary group) rather than $G L_{p}$ and, moreover, the only connected component of the unit element is exploited. The situation is the same as [MR] essentially.

\section{Results}

The results are as follows,

(i) $p=1$ : Kac-Peterson $[\mathrm{KP}]$ (or $[\mathrm{PS}]$ )

$$
\begin{aligned}
\alpha(g, q ; w) & \equiv 0, \\
\eta_{1}(X, Y ; F) & =-\frac{1}{8} \operatorname{tr}[[\varepsilon, X],[\varepsilon, Y]] \varepsilon \\
& =\operatorname{tr}(b(X) c(Y)-b(Y) c(X)) .
\end{aligned}
$$

(ii) $p=2$ : Mickelsson-Rajeev $[\mathrm{MR}]$

$$
\begin{aligned}
\alpha(g, q ; w)= & \exp \left[-\operatorname{tr}\left\{\left(1-q^{-1} a\right)\left(w_{+}-1\right)+q^{-1} b\left(\frac{1}{2} F_{21}-w_{-}\right)\right\}\right], \\
\eta_{2}(X, Y ; F)= & \frac{1}{8} \operatorname{tr}[[\varepsilon, X],[\varepsilon, Y]](F-\varepsilon) \\
= & -\frac{1}{2} \operatorname{tr}\left\{(b(X) c(Y)-b(Y) c(X))\left(F_{11}-1\right)\right. \\
& \left.-b(X)\left(F_{22}+1\right) c(Y)+b(Y)\left(F_{22}+1\right) c(X)\right\} .
\end{aligned}
$$

(iii) $p=3$ :

$$
\alpha(g, q ; w)=\exp \left[\frac{1}{2} \operatorname{tr}\left\{\tilde{\gamma}(g, q ; w)+2\left(q^{-1} a-1\right)\left(\frac{F_{11}-1}{2}\right)^{2}\right\}\right]
$$

where

$$
\begin{aligned}
& \tilde{\gamma}(g, q ; w)=\left\{\left(\left(q^{-1} a-1\right) w_{+}+q^{-1} b w_{-}+w_{+}-1\right)\left(w_{+}^{+}-1\right)\right. \\
& \cdot\left(\left(q^{-1} a-1\right) w_{+}+q^{-1} b w_{-}+w_{+}-1\right)\left(w_{+}^{+}-1\right) \\
&+2\left(\left(q^{-1} a-1\right) w_{+}+q^{-1} b w_{-}+w_{+}-1\right)^{2}\left(w_{+}^{+}-1\right) \\
&+2\left(\left(q^{-1} a-1\right) w_{+}+q^{-1} b w_{-}+w_{+}-1\right)\left(w_{+}^{+}-1\right)^{2} \\
&-\left(w_{+}-1\right)\left(w_{+}^{\dagger}-1\right)\left(w_{+}-1\right)\left(w_{+}^{\dagger}-1\right) \\
&\left.-2\left(w_{+}-1\right)^{2}\left(w_{+}^{+}-1\right)-2\left(w_{+}-1\right)\left(w_{+}^{\dagger}-1\right)^{2}\right\}, \\
& \eta_{3}(X, Y ; F)=\frac{1}{64} \operatorname{tr}\left\{2[[\varepsilon, X],[\varepsilon, Y]](F-\varepsilon)^{3}\right.\left.-[\varepsilon, X](F-\varepsilon)[\varepsilon, Y](F-\varepsilon)^{2}+[\varepsilon, Y](F-\varepsilon)[\varepsilon, X](F-\varepsilon)^{2}\right\} \\
&=\frac{1}{4} \operatorname{tr}\left\{(b(X) c(Y)-b(Y) c(X))\left(F_{11}-1\right)^{2}\right. \\
&+ b(X)\left(F_{22}+1\right)^{2} c(Y)-b(Y)\left(F_{22}+1\right)^{2} c(X) \\
&-b(X)\left(F_{22}+1\right) c(Y)\left(F_{11}-1\right) \\
&+\left.b(Y)\left(F_{22}+1\right) c(X)\left(F_{11}-1\right)\right\} .
\end{aligned}
$$

(iv) $p \geqq 4$ : It is not easy to calculate.

From the above local formula of $\eta_{p}(X, Y ; F)(p=1,2,3)$, we can guess the general formula $\eta_{p}(X, Y ; F)$ as follows: 
(v) p: any natural number

$$
\begin{aligned}
\eta_{p}(X, Y ; F)= & c_{p} \operatorname{tr}\left\{\sum _ { l = 0 } ^ { p - 1 } ( - 1 ) ^ { ( p - 1 ) - l } \left(b(X)\left(F_{22}+1\right)^{l} c(Y)\left(F_{11}-1\right)^{(p-1)-l}\right.\right. \\
& \left.\left.-b(Y)\left(F_{22}+1\right)^{l} c(X)\left(F_{11}-1\right)^{(p-1)-l}\right)\right\},
\end{aligned}
$$

where $c_{p}$ is a constant which only depends on $p$.

In fact, we can show that this satisfies the Jacobi identity (Eq. (49)) (see Appendix). Therefore, $\eta_{p}(X, Y ; F)$ above becomes the two-cocycle. (Of course, $\eta_{p}(X, Y ; F)$ coincides each case (i), (ii), and (iii) with suitable $\left.c_{p}\right)$.

We conjecture that $\eta_{p}$ (Eq. (60)) gives all of the cocycles.

\section{Infinite Charge Renormalization}

We establish the relation $\eta_{p}(X, Y ; F)$ and $\eta_{p+1}(X, Y ; F)$ in this section. Consider the $\eta_{p}(X, Y ; F)$, where $X, Y \in g l_{p+1}$ and $F \in \mathrm{Gr}_{p+1}$. Then this is a two-cocycle but divergent. So we must subtract the divergence in order to get the well-defined two-cocycle: we must find a one-cocycle $\beta_{p}(X ; F)$ such that

$$
\left(\eta_{p}+\delta \beta_{p}\right)(X, Y ; F)=\eta_{p+1}(X, Y ; F)
$$

where

$$
\delta \beta_{p}(X, Y ; F)=\delta_{X} \beta_{p}(Y ; F)-\delta_{Y} \beta_{p}(X ; F)+\beta_{p}([X, Y] ; F) .
$$

In $[\mathrm{MR}](p=1),(61)$ is interpreted as the "infinite charge renormalization." Here we generalize their interpretation to arbitrary $p$. The results are stated as follows:

(i) $p=1[\mathrm{MR}]$

$$
\beta_{1}(X ; F)=\frac{1}{16} \operatorname{tr}[X, \varepsilon][F, \varepsilon]
$$

(ii) $p=2$

$$
\beta_{2}(X, F)=-\frac{1}{64} \operatorname{tr}[X, \varepsilon][F, \varepsilon](F-\varepsilon)^{2} .
$$

We conjecture the form of $\beta_{p}$ for any natural number $p$, (iii) $p$ : any natural number

$$
\beta_{p}(X, F)=d_{p} \operatorname{tr}[X, \varepsilon][F, \varepsilon](F-\varepsilon)^{2(p-1)},
$$

where $d_{p}$ is constant only depending on $p$.

\section{The Highest Weight Vector and State Vectors}

We define holomorphic cross sections of Det* (the dual bundle of Det ${ }_{p}$ ). These are identified with functions

$$
\Psi: \mathrm{St}_{p} \rightarrow C, \quad \Psi(w t)=\Psi(w) \omega_{p}\left(w_{+}, t\right) .
$$

For example, $\Psi_{0}(w)=\operatorname{det}_{p} w_{+}$, which is the "highest weight vector" [MR]. Let $\Gamma\left(\operatorname{Det}_{p}^{*}\right)$ be the set of all cross sections $\Psi$. 
We define $(i)=\left\{i_{1}, i_{2}, \ldots, i_{n}, \ldots\right\} \in N$, which is any finite set [M], and put $S=\{(i)\}$.

Then we define the matrix $w(i)$ by exchanging the rows labeled by $(i)$ of $w_{+}$for the corresponding rows of $w_{-}$:

$$
w(i)=\left(\begin{array}{c}
w_{+}^{(1)} \\
\vdots \\
w_{-}^{\left(i_{1}\right)} \\
\vdots \\
w_{-}^{\left(i_{n}\right)} \\
\vdots
\end{array}\right) .
$$

It is trivial that $w(i)-w_{+} \in I_{1}$ and $w(i)=w_{+}$if (i) is the empty set.

Now we define

$$
\Psi_{(i)}(w) \stackrel{\text { def. }}{=} \operatorname{det}_{p} w(i) \cdot e^{\alpha_{p}(w(i), w+)} .
$$

Since $\Psi_{(i)}(w)$ should satisfy $\Psi_{(i)}(w t)=\Psi_{(i)}(w) \cdot \omega_{p}\left(w_{+}, t\right)$, we have

$$
\gamma_{p}(w(i), t)-\gamma_{p}\left(w_{+}, t\right)=-\left\{\alpha_{p}\left(w(i) t, w_{+} t\right)-\alpha_{p}\left(w(i), w_{+}\right)\right\},
$$

see Eqs. (21)-(23).

We state our result.

(i) $p=2[\mathrm{M}]$,

$$
\Psi_{(i)}(w)=\operatorname{det}_{2} w(i) \cdot e^{\operatorname{tr}(w(i)-w+)} .
$$

(ii) $p=3$,

$$
\Psi_{(i)}(w)=\operatorname{det}_{3} w(i) \cdot e^{\left.-\operatorname{tr}((1 / 2) w(l))^{2}-2 w(l)-(1 / 2) w_{+}^{2}+2 w_{+}\right)} .
$$

Therefore, we have shown that $\Psi_{(i)}$ is a holomorphic section for each $(i) \in S$.

We conjecture that $\left\{\Psi_{(i)} \mid(i) \in S\right\}$ is a holomorphic basis of $\Gamma\left(\operatorname{Det}_{p}^{*}\right)$ :

$$
\forall \Psi \in \Gamma\left(\operatorname{Det}_{p}^{*}\right) \Rightarrow \Psi=\sum_{(i)} c_{(i)} \Psi_{(i)}
$$

\section{Discussion}

We note again that all the above discussions were based on the $\hat{U}_{p}$ (the unitary subgroup of $\widehat{G L_{p}}$ ) rather than $\widehat{G L_{p}}$. We want to construct the representation of the $\widehat{G L}_{p}$ (or $\hat{U}_{p}$ ) on a "Hilbert space," (for the general discussion, see [MR]).

We can define an inner product on $\Gamma\left(\operatorname{Det}_{p}^{*}\right)$ as follows:

$$
\left\langle\Psi_{1}, \Psi_{2}\right\rangle=\int_{\mathrm{Gr}_{p}} d m \bar{\Psi}_{1}(w) \Psi_{2}(w) l(w)^{-2},
$$

if the quasi-invariant measure $d m$ on $\mathrm{Gr}_{p}$ exists (in general, for $p \geqq 2$, the measure $d m$ is unknown, but for $p=1$, the measure may be given by [P1]), where

$$
l(w)=\exp \left\{-\frac{1}{2} \gamma_{p}\left(w_{+}, w_{+}^{\dagger}\right)\right\} .
$$


Given the inner product on $\Gamma\left(\right.$ Det $\left._{p}^{*}\right)$, we can construct the representation:

$$
\begin{gathered}
\left.T: \widehat{G L_{p}} \text { (respectively } \widehat{U}_{p}\right) \rightarrow \Gamma\left(\text { Det }_{p}^{*}\right), \\
(T(g, q, \lambda) \Psi)(w)=\lambda\left(g^{-1} F\right)^{-1} \alpha\left(g, q ; g^{-1} w q\right)^{-1} \Psi\left(g^{-1} w q\right) .
\end{gathered}
$$

Then we can shown this representation is unitary.

But unfortunately, Pickrell [P2] has shown that the unitary subgroup of the group extension $\widehat{G L}_{p}(p>1)$ does not have separable Hilbert space representations which are nontrivial on the extension part. So there may be no quasi-invariant measure on $\operatorname{Gr}_{p}(p>1)$.

Last we note that the results of $[\mathrm{MR}]$ and ours are deeply related to "Universal Yang-Mills Theory" proposed by Rajeev [R] and developed by us [TF]. We will discuss this point in another paper.

\section{Appendix}

In this appendix, we show that Eq. (60) satisfies the Jacobi identity (Eq. (49)). First of all, we list some useful formulas,

$$
\begin{aligned}
b([X, Y]) & =a(X) b(Y)-a(Y) b(X)+b(X) d(Y)-b(Y) d(X), \\
c([X, Y]) & =c(X) a(Y)-c(Y) a(X)+d(X) c(Y)-d(Y) c(X), \\
([Z, F])_{11} & =a(Z)\left(F_{11}-1\right)+b(Z) F_{21}-\left(F_{11}-1\right) a(Z)-F_{12} c(Z), \\
([Z, F])_{22} & =c(Z) F_{12}+d(Z)\left(F_{22}+1\right)-F_{21} b(Z)-\left(F_{22}+1\right) d(Z), \\
F_{12}\left(F_{22}+1\right) & =-\left(F_{11}-1\right) F_{12}, \quad F_{21}\left(F_{11}-1\right)=-\left(F_{22}+1\right) F_{21}, \\
Z \cdot\left(F_{11}-1\right)^{n} & =\sum_{k=0}^{n-1}\left(F_{11}-1\right)^{k}(-[Z, F])_{11}\left(F_{11}-1\right)^{(n-1)-k}, \\
Z \cdot\left(F_{22}+1\right)^{n} & =\sum_{k=0}^{n-1}\left(F_{22}+1\right)^{k}(-[Z, F])_{22}\left(F_{22}+1\right)^{(n-1)-k} .
\end{aligned}
$$

Now we consider the Lie derivatives, $Z \cdot \eta_{p}(X, Y ; F)$

$$
\begin{gathered}
=\left.\frac{d}{d t} \eta_{p}\left(X, Y ; e^{-t Z} F e^{t Z}\right)\right|_{t=0}=c_{p} \operatorname{tr}\left\{\sum_{l=0}^{p-1}(-1)^{(p-1)-l}\right. \\
\cdot\left(b(X)\left(\sum_{k=0}^{l-1}\left(F_{22}+1\right)^{k}(-[Z, F])_{22}\left(F_{22}+1\right)^{(l-1)-k}\right) c(Y)\left(F_{11}-1\right)^{(p-1)-l}\right. \\
+b(X)\left(F_{22}+1\right)^{l} c(Y)\left(\sum_{k=0}^{(p-1)-(l-1)}\left(F_{11}-1\right)^{k}(-[Z, F])_{11}\left(F_{11}-1\right)^{(p-1)-k-(l+1)}\right) \\
-c(X)\left(\sum_{k=0}^{(p-1) /(l+1)}\left(F_{11}-1\right)^{k}(-[Z, F])_{11}\left(F_{11}-1\right)^{(p-1)-(l+1)-k}\right) b(Y)\left(F_{22}+1\right)^{l} \\
\left.\left.-c(X)\left(F_{11}-1\right)^{(p-1)-l} b(Y)\left(\sum_{k=0}^{l-1}\left(F_{22}+1\right)^{k}(-[Z, F])_{22}\left(F_{22}+1\right)^{(l-1)-k}\right)\right)\right\}
\end{gathered}
$$




$$
\begin{aligned}
= & c_{p} \operatorname{tr}\left\{\sum _ { l = 0 } ^ { p - 1 } ( - 1 ) ^ { ( p - 1 ) - l } \left(\left(c(X)\left(F_{11}-1\right)^{(p-1)-l} b(Y)-c(Y)\left(F_{11}-1\right)^{(p-1)-l} b(X)\right)\right.\right. \\
& \cdot\left(\sum _ { k = 0 } ^ { l - 1 } ( F _ { 2 2 } + 1 ) ^ { k } \left(c(Z) F_{12}+d(Z)\left(F_{22}+1\right)-F_{21} b(Z)\right.\right. \\
& \left.\left.\left.-\left(F_{22}+1\right) d(Z)\right)\left(F_{22}+1\right)^{(l-1)-k}\right)-b(X)\left(F_{22}+1\right)^{l} c(Y)-b(Y)\left(F_{22}+1\right)^{l} c(X)\right) \\
& \cdot\left(\sum _ { k = 0 } ^ { ( p - 1 ) - ( l - 1 ) } ( F _ { 1 1 } - 1 ) ^ { k } \left(a(Z)\left(F_{11}-1\right)+b(Z) F_{21}-\left(F_{11}-1\right) a(Z)\right.\right. \\
& \left.\left.\left.\left.-F_{12} c(Z)\right)\left(F_{11}-1\right)^{(p-1)-(l+1)-k}\right)\right)\right\} .
\end{aligned}
$$

Using (a3)-(a8), we have following results after simple but tedious calculations:

$$
\begin{aligned}
Z \cdot \eta_{p} & (X, Y ; F) \\
= & c_{p} \operatorname{tr}\left\{\sum _ { l = 0 } ^ { p - 1 } ( - 1 ) ^ { ( p - 1 ) - l } \left((a(Z) b(X)-b(X) d(Z))\left(F_{22}+1\right)^{l} c(Y)\left(F_{11}-1\right)^{(p-1)-l}\right.\right. \\
& -(a(Z) b(Y)-b(Y) d(Z))\left(F_{22}+1\right)^{l} c(X)\left(F_{11}-1\right)^{(p-1)-l} \\
& +(c(X) a(Z)-d(Z) c(X))\left(F_{11}-1\right)^{(p-1)-l} b(Y)\left(F_{22}+1\right)^{l} \\
& -(c(Y) a(Z)-d(Z) c(Y))\left(F_{11}-1\right)^{(p-1)-l} b(X)\left(F_{22}+1\right)^{l} \\
& +\sum_{k=0}^{(p-1)-l-1}\left(P_{k}(Z, X, Y)-P_{k}(X, Y, Z)+P_{k}(Y, X, Z)-P_{k}(Z, Y, X)\right. \\
& \left.\left.\left.+Q_{k}(Z, X, Y)-Q_{k}(X, Y, Z)+Q_{k}(Y, X, Z)-Q_{k}(Z, Y, X)\right)\right)\right\}
\end{aligned}
$$

where

$$
\begin{aligned}
& P_{k}(X, Y, Z)=\left(F_{11}-1\right)^{(p-1)-l-1-k} b(X)\left(F_{22}+1\right)^{l} c(Y)\left(F_{11}-1\right)^{k} b(Z) F_{21}, \\
& Q_{k}(X, Y, Z)=c(X)\left(F_{11}-1\right)^{(p-1)-l-1-k} b(Y)\left(F_{22}+1\right)^{l} c(Z)\left(F_{11}-1\right)^{k} F_{12},
\end{aligned}
$$

The others are given by the cyclic permutations of $X, Y$, and $Z$. Using the fact that $P_{k}(Z, X, Y)+($ cyclic permutations $)+Q_{k}(Z, X, Y)+($ cyclic permutations $)=0, \quad(\mathrm{a} 13)$ we have,

$$
\begin{aligned}
Z \cdot \eta_{p} & (X, Y ; F)+X \cdot \eta_{p}(Y, Z ; F)+Y \cdot \eta_{p}(Z, X ; F) \\
= & c_{p} \operatorname{tr}\left\{\sum_{l=0}^{p-1}(-1)^{(p-1)-l}\right. \\
& \cdot\left((a(X) b(Y)-a(Y) b(X)+b(X) d(Y)-b(Y) d(X))\left(F_{22}+1\right)^{l} c(Z)\left(F_{11}-1\right)^{(p-1)-l}\right. \\
& +(a(Y) b(Z)-a(Z) b(Y)+b(Y) d(Z)-b(Z) d(Y))\left(F_{22}+1\right)^{l} c(X)\left(F_{11}-1\right)^{(p-1)-l} \\
& +(a(Z) b(X)-a(X) b(Z)+b(Z) d(X)-b(X) d(Z))\left(F_{22}+1\right)^{l} c(Y)\left(F_{11}-1\right)^{(p-1)-l}
\end{aligned}
$$




$$
\begin{aligned}
& -(c(X) a(Y)-c(Y) a(X)+d(X) c(Y)-d(Y) c(X))\left(F_{11}-1\right)^{(p-1)-l} b(Z)\left(F_{22}+1\right)^{l} \\
& -(c(Y) a(Z)-c(Z) a(Y)+d(Y) c(Z)-d(Z) c(Y))\left(F_{11}-1\right)^{(p-1)-l} b(X)\left(F_{22}+1\right)^{l} \\
& \left.\left.-(c(Z) a(X)-c(X) a(Z)+d(Z) c(X)-d(X) c(Z))\left(F_{11}-1\right)^{(p-1)-l} b(Y)\left(F_{22}+1\right)^{l}\right)\right\} \\
= & c_{p} \operatorname{tr}\left\{\sum _ { l = 0 } ^ { p - 1 } ( - 1 ) ^ { ( p - 1 ) - l } \left(b([Z, Y])\left(F_{22}+1\right)^{l} c(Z)\left(F_{11}-1\right)^{(p-1)-l}\right.\right. \\
& +b([Y, Z])\left(F_{22}+1\right)^{l} c(X)\left(F_{11}-1\right)^{(p-1)-l} \\
& +b([Z, X])\left(F_{22}+1\right)^{l} c(Y)\left(F_{11}-1\right)^{(p-1)-l} \\
& -c([X, Y])\left(F_{11}-1\right)^{(p-1)-l} b(Z)\left(F_{22}+1\right)^{l} \\
& -c([Y, Z])\left(F_{11}-1\right)^{(p-1)-l} b(X)\left(F_{22}+1\right)^{l} \\
& \left.\left.-c([Z, X])\left(F_{11}-1\right)^{(p-1)-l} b(Y)\left(F_{22}+1\right)^{l}\right)\right\} .
\end{aligned}
$$

Since $b(), c() \in I_{2 p}$ and $F_{11}-1, F_{22}+1 \in I_{p}$, we can easily see that each term of (a14) is in trace class, and we can modify (a14) as follows:

$$
\begin{aligned}
Z \cdot \eta_{p}(X, Y ; F)+X \cdot \eta_{p}(Y, Z ; F)+Y \cdot \eta_{p}(Z, X ; F) \\
=c_{p} \operatorname{tr}\left\{\sum _ { l = 0 } ^ { p - 1 } ( - 1 ) ^ { ( p - 1 ) - l } \left(b([X, Y])\left(F_{22}+1\right)^{l} c(Z)\left(F_{11}-1\right)^{(p-1)-l}\right.\right. \\
\left.\quad-c([X, Y])\left(F_{11}-1\right)^{(p-1)-l} b(Z)\left(F_{22}+1\right)^{l}\right\} \\
\quad+c_{p} \operatorname{tr}\left\{\sum _ { l = 0 } ^ { p - 1 } ( - 1 ) ^ { ( p - 1 ) - l } \left(b([Y, Z])\left(F_{22}+1\right)^{l} c(X)\left(F_{11}-1\right)^{(p-1)-l}\right.\right. \\
\left.\left.\quad-c([Y, Z])\left(F_{11}-1\right)^{(p-1)-l} b(X)\left(F_{22}+1\right)^{l}\right)\right\} \\
+c_{p} \operatorname{tr}\left\{\sum _ { l = 0 } ^ { p - 1 } ( - 1 ) ^ { ( p - 1 ) - l } \left(b([Z, X])\left(F_{22}+1\right)^{l} c(Y)\left(F_{11}-1\right)^{(p-1)-l}\right.\right. \\
\left.\left.\quad-c([Z, X])\left(F_{11}-1\right)^{(p-1)-l} b(Y)\left(F_{22}+1\right)^{l}\right)\right\} \\
=\eta_{p}([X, Y], Z ; F)+\eta_{p}([Y, Z], X ; F)+\eta_{p}([Z, X], Y ; F) .
\end{aligned}
$$

Thus we have just the Jacobi identity (Eq. (49)).

Acknowledgements. We would like to thank Profs. Shoichiro Otsuki and Masahiro Imachi for a careful reading of the manuscript. We also thank the referee for useful suggestions.

\section{References}

[F] Faddeev, L.: Operator anomaly for the gauss law. Phys. Lett. 145B, 81-84 (1984)

[S] Simon, B.: Trace ideals and their applications. Cambridge: Cambridge University Press 1979 
[C] Connes, A.: Non-commutative differential grometry. I.H.E.S. Publ. Math. 62, 41-144 (1985)

[MR] Mickelsson, J., Rajeev, S. G.: Current algebras in $d+1$-dimensions and determinant bundles over infinite-dimensional Grassmannians. Commun. Math. Phys. 116, 365-400 (1988)

[KP] Kač, V. G., Peterson, D. H.: Lectures on the infinite wedge representation and the MPP hierarchy. Proceedings of the Summer School on Completely Integrable Systems Montreal, Canada, August, (1985)

[PS] Pressley, A., Segal, G.: Loop groups. Oxford, UK: Oxford University Press 1986

[M] Mickelsson, J.: Current algebra representation for the $3+1$ dimensional Dirac-Yang-Mills theory. Commun. Math. Phys. 117, 261-277 (1988)

[P1] Pickrell, D.: Measures on infinite-dimensional Grassmann manifolds. J. Funct. Anal. 70, 323-356 (1987)

[P2] Pickrell, D.: On the Mickelsson-Faddeev extension and unitary representations. Commun. Math. Phys. 123, 617-625 (1989)

[R] Rajeev, S. G.: An exactly integrable algebraic model for $(3+1)$-dimensional Yang-Mills theory. Phys. Lett. B209, 53-58 (1988)

[TF] Tanaka, M., Fujii, K.: Note on algebraic analogue of Yang-Mills-Higgs theory. (preprint) KYUSHU-88-HE-7

Communicated by L. Alvarez-Gaumé

Received July 25, 1989; in revised form November 13, 1989 